\title{
Pharmacological Activation of Thermo-Transient Receptor Potential Vanilloid 3 Channels Inhibits Hair Growth by Inducing Cell Death of Hair Follicle Outer Root Sheath
}

\author{
Kangjiao Yan, ${ }^{1}$ Xiaoying Sun, ${ }^{1}$ Gongxin Wang, Yani Liu, and KeWei Wang \\ Department of Pharmacology, School of Pharmacy, Qingdao University, Qingdao, China \\ Received March 13, 2019; accepted May 30, 2019
}

\begin{abstract}
Hair growth starts from hair follicles that reside in dermis, and abnormal hair growth is an early sign of hair follicle disease or systemic illness such as alopecia or hair loss. Therefore, identifying a target critical for dysfunctional hair follicles is fundamental to alleviating dermatologic or systemic diseases with hair abnormalities. The warm temperature-activated $\mathrm{Ca}^{2+}-$ permeable transient receptor potential vanilloid 3 (TRPV3) channel protein is abundantly expressed in the skin keratinocytes, and dysfunctional TRPV3 causes human congenital Olmsted syndrome, characterized by skin diseases and alopecia, indicating an important role for TRPV3 in hair follicle development and hair growth. To validate TRPV3 as a therapeutic target, we investigated the impact of pharmacological modulation of TRPV3 on hair growth using a combination of biochemical and cell biology, immunohistochemical, whole-cell patch clamp, RNA interference,
\end{abstract}

and pharmacological approaches. We found that functional TRPV3 channel proteins are highly expressed in hair follicle outer root sheath (ORS) cells as detected by Western blot analysis, immunohistochemical staining, and electrophysiological techniques. Pharmacological activation of TRPV3 by agonist natural carvacrol induces cell death of ORS cells, and topical application of carvacrol to mouse dorsal skin also inhibits hair growth. Conversely, specific inhibition of TRPV3 by inhibitor natural forsythoside $B$ and short-hairpin RNA reverses the cell death induced by carvacrol-mediated TRPV3 activation in human ORS cells. Furthermore, forsythoside $B$ results in a significant reversal of hair growth inhibition induced by agonist carvacrol. Altogether, our findings demonstrate that TRPV3 channel is critical for regulation of hair growth, and inhibition of TRPV3 may represent a promising therapy for hair loss or hair follicle-related skin diseases.

\section{Introduction}

The hair follicle residing in the dermal layer of the skin is a tunnel-like segment of the epidermis and grows different types of hair. Abnormalities of hair growth and appearance are early signs of dysfunctional hair follicles, including well known alopecia or hair loss or systemic diseases (Spencer and Callen, 1987; Sperling, 2001). Within the hair growth cycle there are three sequential phases: anagen, catagen, and telogen (Paus, 1998; Müller-Röver et al., 2001). The anagen, also known as the growth phase, is characterized by the change of skin pigmentation that is considered as a transition from telogen phase to anagen phase (Slominski and Paus, 1993; Slominski et al., 1994; Müller-Röver et al., 2001). Hair follicle as a dynamic organ is a feature of outer root sheath (ORS) cells that are essentially a stratified epithelium contiguous with the epidermis. The hair follicle surrounding the hair

This project was supported by grants awarded to K.W. from National Natural Sciences Foundation of China [81573410] and the Ministry of Science and Technology of China [2018ZX09711001-004-006], and a grant awarded to X.S. from Shandong Provincial Natural Science Foundation of China [ZR2017BH020].

${ }^{1}$ K.Y. and X.S. contributed equally to this work

https://doi.org/10.1124/jpet.119.258087. fiber and inner root sheath also serves as an immunologic sentinel and a sensory organ of the skin (Paus and Cotsarelis, 1999), and it has been suggested that ORS cells are fundamental for maintaining the intact structure of hair follicles and regulating hair growth (Paus and Cotsarelis, 1999). Therefore, identifying a target critical for functional and dysfunctional hair follicle is important for diagnosis and treatment of dermatologic or systemic diseases with hair abnormalities.

The warm temperature-activated transient receptor potential vanilloid 3 (TRPV3) channel is immunostained in the ORS cells of hair follicles (Borbíró et al., 2011), suggesting a role for the channel in regulation of hair growth. Temperature is a significant factor for regulation of animal hair growth in a natural environment (Kunz et al., 2009; Chen and Chuong, 2012). The nonselective cation $\mathrm{Ca}^{2+}$-permeable TRPV 3 channel is activated by temperatures from warm $\left(>33^{\circ} \mathrm{C}\right)$ to hot (Clapham, 2003; Jordt et al., 2003), and is abundantly expressed in the keratinocytes (Peier et al., 2002; Chung et al., 2004b) and hair apparatus (Borbiro et al., 2011). TRPV3 participates in numerous physiologic processes, such as skin barrier formation, cutaneous sensation, and hair growth (Cheng et al., 2010; Aijima et al., 2015; Wang and Wang, 2017). In rodents, the spontaneous gain-of-function mutation (Gly573 substituted by Ser or Gly573 substituted by Cys) of

ABBREVIATIONS: ANOVA, analysis of variance; FB, forsythoside B; H\&E, hematoxylin and eosin; HEK, human embryonic kidney; MTT, 3-(4,5-; -2-yl)-2,5-di, tetrazolium bromide; ORS, out root sheath; sh, short-hairpin; TRPA1, transient receptor potential ankyrin 1; TRPV3, transient receptor potential vanilloid 3; TRPV4, transient receptor potential vanilloid 4. 
the TRPV3 gene causes hair loss and AD-like dermatitis in both DS-Nh mice and WBN/Kob-Ht rats (Asakawa et al., 2006; Steinhoff and Biro, 2009). In humans, we and others identified the gain-of-function mutations (W692G, G573C and G573S) the TRPV3 gene that cause Olmsted syndrome, a rare congenital disorder characterized by skin inflammatory diseases such as palmoplantar intense itch, and alopecia in most cases (Lai-Cheong et al., 2012; Lin et al., 2012; Choi et al., 2018). Conversely, TRPV3-deficient mice exhibit phenotypes of curly whiskers, a thin cuticle, wavy hair, and misaligned hair follicles (Moqrich et al., 2005; Schneider et al., 2008; Cheng et al., 2010), indicating a critical role for TRPV3 in normal morphogenesis of hair and hair follicles.

The polymodal TRPV3 channel is also activated by several natural compounds, such as carvacrol, camphor, isoborneol, and thymol (Chung et al., 2004a; Xu et al., 2006; Bang et al., 2010; Cao et al., 2012; Cui et al., 2018). Carvacrol (5-isopropyl2-methylphenol), a major active component of oregano, activates TRPV3 with an $\mathrm{EC}_{50}$ value $0.49 \pm 0.07 \mathrm{mM}$ (Vogt-Eisele et al., 2007). Carvacrol $(250 \mu \mathrm{M})$ also activates transient receptor potential ankyrin 1 (TRPA1) current that is quickly desensitized in the continuous presence of this agonist (Xu et al., 2006; Cui et al., 2018), whereas TRPV1 and transient receptor potential vanilloid 3 (TRPV4) are insensitive to carvacrol (1 mM) (Xu et al., 2006). In TRPV3 agonist carvacrol-induced scratching, knocking out TRPV3 suppresses scratching behavior (Cui et al., 2018). Silencing TRPV3 also can reverse the cell death or $\mathrm{Ca}^{2+}$ signals induced by carvacrol in normal human epidermal keratinocytes and human sebocytes (Szöllősi et al., 2018; Szántó et al., 2019). These results indicate the specificity of carvacrol in activation of TRPV3, which plays a critical role in itching. However, there is a significant lack of specific TRPV3 blockers. At present, TRPV3 can only be selectively inhibited by natural forsythoside $\mathrm{B}$ (FB), an active ingredient of Lamiophlomis rotate plant leaves (Zhang et al., 2019).

In this study, we investigated the effect in mice of pharmacological modulation of TRPV3 channel function on hair growth using a combination of biochemical and cell biology, immunohistochemical, and pharmacological approaches. Our findings show that TRPV3 is robustly expressed in ORS cells of hair follicle, and pharmacological activation of TRPV3 by agonist carvacrol results in a time- and concentrationdependent delay of hair growth by decreasing hair follicles and inhibiting hair shaft elongation. Conversely, inhibition of TRPV3 by forsythoside B can significantly reverse the hair growth inhibition induced by carvacrol. Our findings demonstrate that topical application of TRPV3 channel modulators may hold a promising therapy for treatment of either hair loss or growth and dysfunctional hair follicle-related diseases.

\section{Materials and Methods}

Animals. Male C57BL/6 mice (7 to 8 weeks) were obtained from Beijing Vital River Laboratory. Mice were housed for at least 1 week to adapt to their environment controlled at a temperature of $23 \pm 2^{\circ} \mathrm{C}$ and a 12-hour light-dark cycle. For the establishment of carvacrolinduced hairless model, mouse dorsal skin $(2.5 \times 3.5 \mathrm{~cm})$ was depilated with electric hair clipper and depilatory paste 24 hours before the start of an experiment. All experiments were approved by the Animal Care and Use Committee of Qingdao University Health Science Center.
Drugs. Natural monoterpenoid phenol carvacrol (5-isopropyl-methylphenol) (MW 150.2) was obtained from Sigma-Aldrich Company and stored at $4^{\circ} \mathrm{C}$. Natural compound forsythoside B (MW 756.7) was purchased from Shanghai Taoto Biotech Corporation, Ltd. Forsythoside B (100 mM as stock) was dissolved in dimethyl sulfoxide. For whole-cell patch clamp-recording experiments, compounds were diluted in perfusion solution. For cell death and MTT assays, compounds were prepared using cell culture medium. For in vivo experiments, all different concentrations $(0.3,1,3 \%$ equivalent to $19.1,63.7$, and $191.1 \mathrm{mM})$ of TRPV3 agonist carvacrol were prepared in 50\% ethanol and locally applied to the shaved dorsal area in $150 \mu$ l. The different concentrations $(0.3,1,3 \mathrm{mM})$ of TRPV3 antagonist forsythoside B were prepared in normal saline and intradermally injected into the modeling area in $50 \mu \mathrm{l}$. For in vitro or in vivo experiments, serial dilutions of compounds with buffer were made with a final concentration of dimethyl sulfoxide less than $0.1 \%$ or $3 \%$, respectively.

Cell Culture. Human embryonic kidney (HEK)293 cells were cultured in Dulbecco's modified Eagle's medium with $10 \%$ fetal bovine serum under the conditions of $5 \% \mathrm{CO}_{2}$ and $37^{\circ} \mathrm{C}$. HEK293 cells were transiently transfected with hTRPV $3 \mathrm{cDNA}$ plasmids tagged with green fluorescent protein using Lipofectamine 2000 (Invitrogen, Carlsbad, CA). The human hair outer root sheath cells isolated from scalps were purchased from ScienCell Laboratories (ScienCell). ORS cells were maintained in a culture of mesenchymal stem cell medium in an incubator under conditions of $5 \% \mathrm{CO}_{2}$ and $37^{\circ} \mathrm{C}$. HEK293 cells and ORS cells were seeded on glass coverslips for whole-cell patch clamp recordings.

Western Blot. Lysates of ORS cells were subjected to SDS-PAGE separation before transfer to polyvinylidene fluoride (PVDF) membrane for Western blotting, and first probed with anti-TRPV3 antibody (1:1000; Abcam) followed by secondary anti-rabbit IgG antibody (1:5000; Abcam). The protein bands in the PVDF membrane were detected using a SuperSignal West Pico PLUS Chemiluminescent Substrate (Thermo Scientific).

Immunohistochemistry. Skin tissue sections were incubated using anti-TRPV3 antibody (1:100; Abcam) at $4^{\circ} \mathrm{C}$ overnight and subsequently incubated with secondary anti-rabbit IgG antibody (1:2000; Abcam) for 30 minutes at room temperature before further treatment with streptavidin peroxidase complex and staining with chromogen.

Whole-Cell Patch Clamp Recordings. Whole-cell patch clamp recordings were carried out using a HEKA EPC10 amplifier with PatchMaster software (HEKA; Instrument Inc, Lambrecht/Pfalz, Germany). Patch pipettes were pulled from borosilicate glass using a puller (DMZ-Universal; Zeitz-Instruments $\mathrm{GmbH}$, Martinsried, Germany) and fire polished to a resistance of 3-5 M 2 . For ramp recordings of HEK293 cells, both pipette solution and bath solution contained $130 \mathrm{mM} \mathrm{NaCl}, 0.2 \mathrm{mM}$ EDTA, and $3 \mathrm{mM} \operatorname{HEPES}(\mathrm{pH}$ 7.2). For ramp recordings of ORS cells, the bath solution contained $139 \mathrm{mM}$ $\mathrm{NaCl}, 5 \mathrm{mM} \mathrm{KCl}, 10 \mathrm{mM}$ HEPES, $10 \mathrm{mM}$ glucose, $2 \mathrm{mM} \mathrm{MgCl}_{2}$, and $1.8 \mathrm{mM} \mathrm{CaCl}_{2}, \mathrm{pH} 7.4$ with $\mathrm{NaOH}$. The recording pipette was filled with a solution containing $120 \mathrm{mM} \mathrm{CsCl}, 5 \mathrm{mM}$ EGTA, $10 \mathrm{mM}$ HEPES, $2 \mathrm{mM} \mathrm{CaCl}_{2}, 0.3 \mathrm{mM} \mathrm{Na}-\mathrm{GTP}, 2 \mathrm{mM} \mathrm{Mg}$-ATP, and $8 \mathrm{mM} \mathrm{NaCl}, \mathrm{pH}$ 7.3 with $\mathrm{CsOH}$. Membrane potential was held at $0 \mathrm{mV}$ and current was recorded in response to 500-millisecond voltage ramps from -100 to $+100 \mathrm{mV}$. Current amplitude was analyzed at $\pm 80 \mathrm{mV}$. All data were analyzed using Origin 8.6 software (OriginLab, Northampton, MA).

Cell Death Assay. The cultured ORS cells of human hair follicles were divided into different groups in a six-well plate. All cell nuclei were fluorescently stained with blue dye of Hoechst 33258 (Thermo Fisher Science), and the nuclei of dead cells were labeled with red fluorescent propidium iodide (PI) (Thermo Fisher Science) after treatment with different compounds for 12 hours. Images were randomly collected under the microscope (ECLIPSE Ti-S; Nikon, Tokyo) from each group and were counted automatically using a confocal microscopy (A1R MP; Nikon). The average of PI-stained cells over cells stained by Hoechst dye from five images represented the cell death ratio.

RNA Interference. TRPV3 short-hairpin (sh)RNAs (Y5919, Y5920, and Y5921) were purchased from Obio Technology Corporation 
A

HEK293- hTRPV3

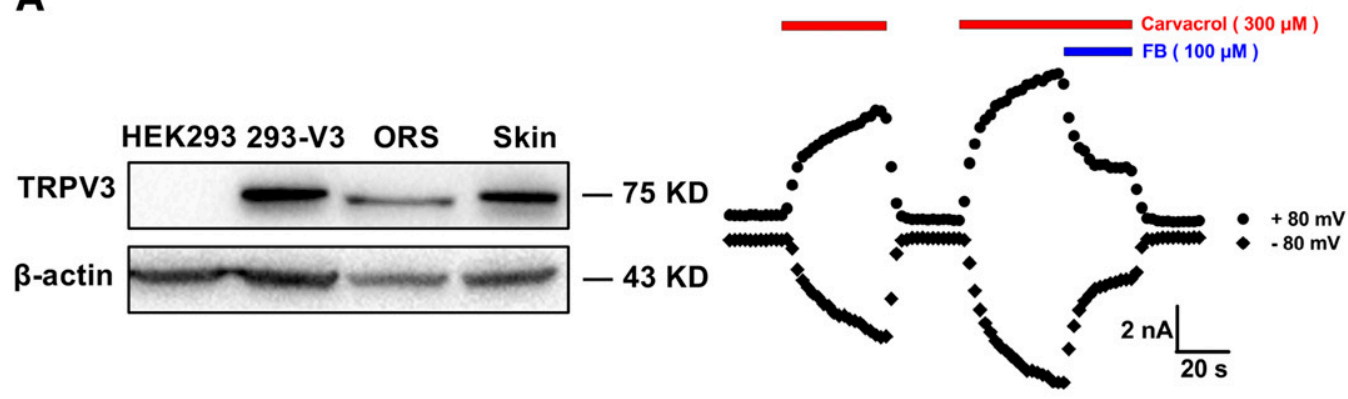

B

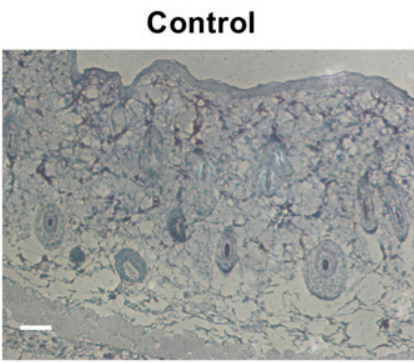

Skin keratinocytes
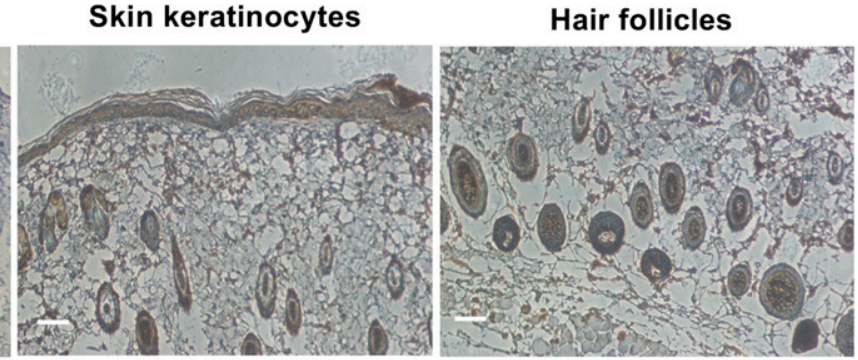

C

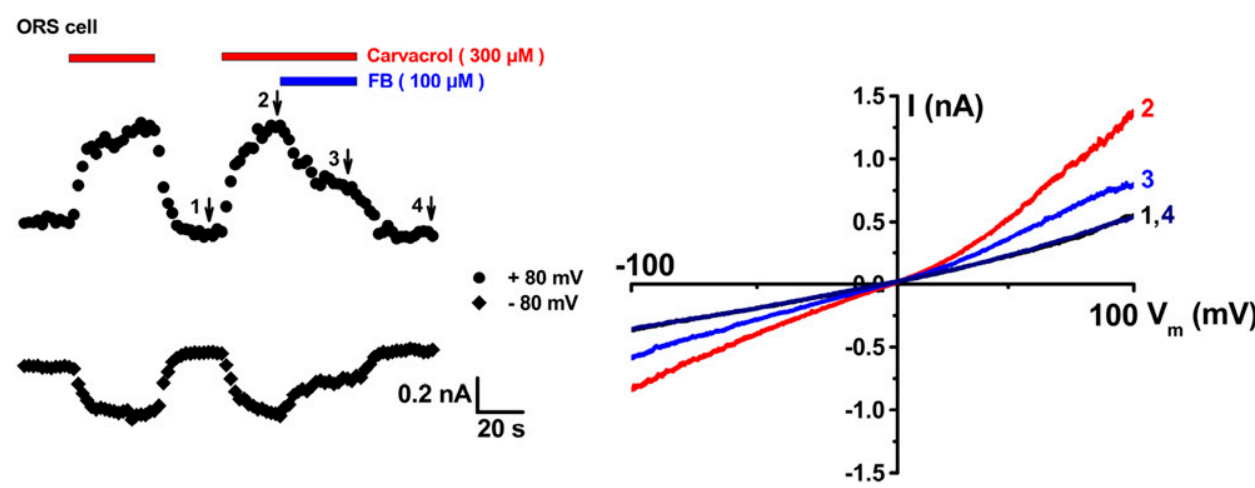

Fig. 1. Functional expression of TRPV3 proteins in mouse skin keratinocytes and ORS cells of hair follicles. (A) Left panel, Western blot analysis of TRPV3 proteins in human outer root sheath cells, dorsal skin of C57BL/6 mice, and HEK293/TRPV3 stably expressing the TRPV3 as positive controls, and untransfected HEK293 cells as negative control. Right panel, whole-cell patch clamp recordings of HEK293 cells overexpressing hTRPV3 in response to carvacrol alone $(300 \mu \mathrm{M}$, red bar) or coapplication of $100 \mu \mathrm{M}$ forsythoside B (blue bar). (B) Immunohistochemistry staining of TRPV3 proteins in mouse skin keratinocytes and hair follicles. Scale bar, $100 \mu \mathrm{m}$. (C) TRPV3 current in ORS cells was activated by $300 \mu \mathrm{M}$ carvacrol (red bar) and inhibited by coapplication of $100 \mu \mathrm{M}$ forsythoside B (blue bar). The right panel shows current-voltage curves of hTRPV3 channel in response to voltage ramps from - 100 to $+100 \mathrm{mV}$ before (1), and after $300 \mu \mathrm{M}$ carvacrol (2), and coaddition of $300 \mu \mathrm{M}$ carvacrol and $100 \mu \mathrm{M}$ forsythoside B (3), and washout (4).

Ltd. (Shanghai, China) for transfection of ORS cells using Lipofectamine 2000 Transfection Reagent (Invitrogen). After 24 hours of transfection, Western blotting was carried out for confirmation of gene knockout efficiency and the most effective short-hairpin (sh)RNA was selected for subsequent experiments.

MTT Assay. ORS cells were cultured in 96-well plates, and the tetrazolium salt MTT for cell viability staining was added after treatment with carvacrol for 24 hours. Formazan dyes from the reduction of tetrazolium salts by mitochondrial dehydrogenases were detected for measurement of cell viability, and the number of viable cells, by absorbance at $490 \mathrm{~nm}$.

Hair Length Measurements. Regrown hairs were randomly plucked from shaved dorsal areas of mouse skin at time points of days $9,12,15,18$, and 21 . The average hair length from 10 hairs per mouse was manually measured.

Histology. Dorsal skin was excised after topical applications of TRPV3 pharmacological modulators (carvacrol or forsythoside B), and maintained in $4 \%$ paraformaldehyde at $4{ }^{\circ} \mathrm{C}$ overnight before being embedded in paraffin blocks for further transverse and longitudinal sectioning at about 5 - to $10-\mu \mathrm{m}$ thickness. The tissue sections were stained with hematoxylin and eosin $(\mathrm{H} \& \mathrm{E})$. Images of tissue sections were selected and photographed under a microscope (ECLIPSE Ti-S; Nikon).

Hair Follicle Counting. Five randomly selected areas $\left(0.09 \mathrm{~mm}^{2}\right.$ per area) from each tissue section stained with $\mathrm{H} \& \mathrm{E}$ were manually counted for an average number of hair follicles. Images of H\&Estained tissue sections were examined and photographed under the microscope (ECLIPSE Ti-S; Nikon).

Data Analysis. All data were expressed as the mean \pm S.D. For two-group comparison statistical significance was assessed by Student's $t$ test (two-tailed, unpaired), and multiple-comparison test was assessed by one-way analysis of variance (ANOVA) or two-way ANOVA followed by Dunnett's test, Bonferroni's test, or Sidak's test using GraphPad Prism 7.0 software. A value of $P<0.05$ was considered statistically significant.

\section{Results}

Functional Expression of TRPV3 Channel in the Skin Keratinocytes and Hair Follicle Outer Root Sheath Cells. To recapitulate TRPV3 expression in the skin keratinocytes (Paus and Cotsarelis, 1999; Stenn and Paus, 2001) 
A
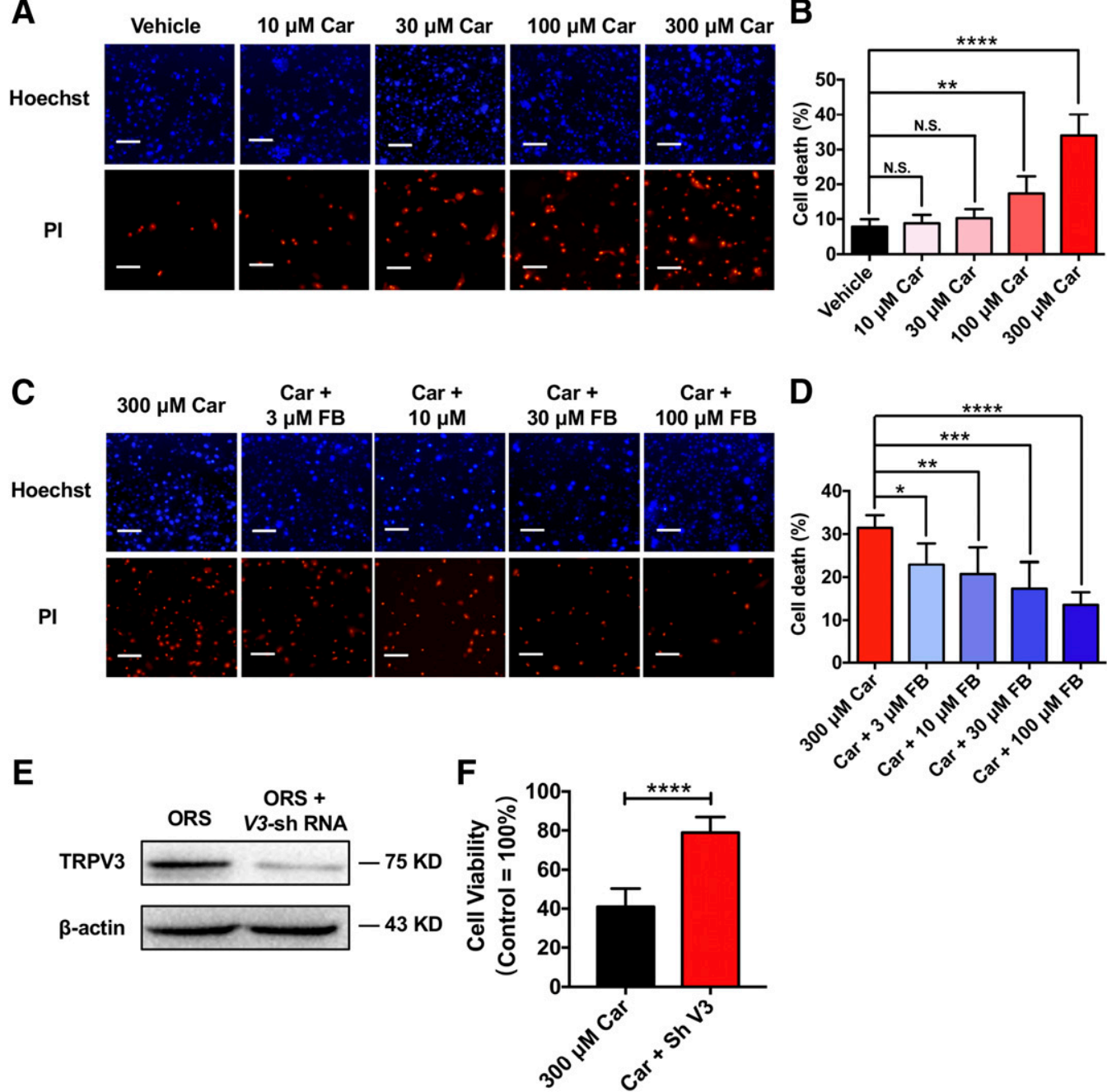

Fig. 2. Inhibition of TRPV3 by forsythoside B attenuates carvacrol-induced ORS cell death. (A) Measurement of cell death for ORS cells before and after treatment of agonist carvacrol in different concentrations for 12 hours in cell death assay. Scale bar, $100 \mu \mathrm{m}$. (B) Summary from (A) for cell death ratio before and after treatment of carvacrol in different concentrations (N.S., no significance; $* * P<0.01$; $* * * * P<0.0001$, by one-way ANOVA, followed by Dunnett's test). (C) Measurement of cell death for ORS cells before and after treatment of carvacrol ( $300 \mu \mathrm{M})$ and different concentrations of forsythoside B for 12 hours in cell death assay. Scale bar, $100 \mu \mathrm{m}$. (D) Summary of (C) for cell death ratio after treatment of carvacrol (300 $\mu$ M) and forsythoside B in different concentrations. Data are presented as the mean \pm S.D. (N.S., no significance, ${ }^{*} P<0.05 ; * * P<0.01 ; * * * P<0.001$; $*_{* * *} P<0.0001$, by one-way ANOVA, followed by Dunnett's test.) (E) Western blot analysis of TRPV3 proteins in ORS cells and ORS cells transfected with TRPV3 shRNA. (F) ORS cells and ORS cells transfected with TRPV3 shRNA were treated with $300 \mu \mathrm{M}$ carvacrol for 24 hours. Cell viability was determined by colorimetric MTT cell viability assay. Data are presented as the mean \pm S.D. $[n=6$, **** $P<0.0001$, by Student's $t$ test (unpaired).]

and ORS cells of hair follicles (Borbiro et al., 2011), we started examining TRPV3 protein expressions in the skin keratinocytes from C57BL/6 mice and primary ORS cells of hair follicles using Western blot assay. As shown in Fig. 1A, left panel, a sharp band of TRPV3 proteins was detected in both primary culture of ORS cells and mouse dorsal skin, compared with untransfected or HEK293 cells transiently expressing TRPV3 currents that had been activated by agonist carvacrol and inhibited by antagonist forsythoside B (Fig. 1A, right panel). Immunohistochemistry staining further confirmed that TRPV3 proteins were mainly distributed in the skin keratinocytes and hair follicles of mouse dorsal skin (Fig. 1B). Whole-cell patch clamp recordings of ORS cells further confirmed that TRPV3 current was also activated by repeated applications of carvacrol $(300 \mu \mathrm{M})$, and TRPV3 current was inhibited by forsythoside B $(100 \mu \mathrm{M})$ about $56.5 \% \pm 6.8 \%$, and the effect could be washed out (Fig. 1C). These results demonstrated that hair follicle ORS cells indeed could express the functional TRPV3 channel, suggesting its role in hair growth. These results are also consistent with TRPV3 expression in human hair folliclederived ORS keratinocytes (Borbiro et al., 2011).

Activation of TRPV3 by Agonist Carvacrol Induces Death of Hair Follicle ORS Cells. TRPV3 agonist carvacrol induces cell death in HEK293 cells expressing TRPV3 channels, HaCaT cells, human epidermal keratinocytes, and human sebocytes (Szöllősi et al., 2018; Zhang et al., 2019). To further confirm the functional expression of TRPV3 in ORS cells, we also examined the effect of TRPV3 activation by carvacrol on human hair follicle ORS cells. As shown in Fig. 2, $\mathrm{A}$ and $\mathrm{B}$, ORS cells treated with carvacrol at different concentrations (10-300 $\mu \mathrm{M})$ for 12 hours exhibited dosedependent increases in cell death ratio in cell death assay, compared with vehicle. In contrast, adding different concentrations of forsythoside $\mathrm{B}(3-100 \mu \mathrm{M})$ in the presence of 
A
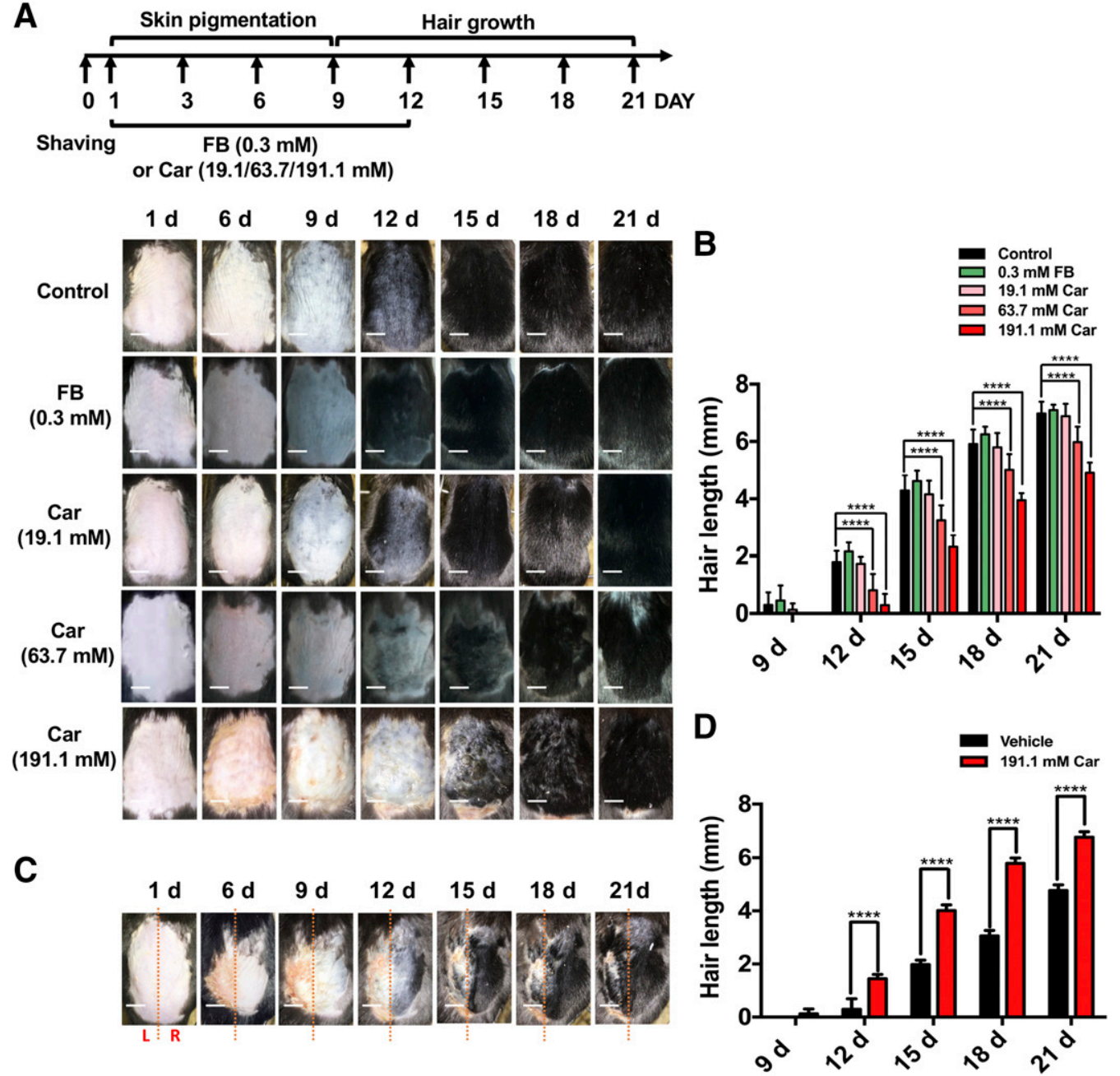

Fig. 3. Pharmacological activation of TRPV3 by carvacrol inhibits hair growth. (A) Top panel, a schematic drawing for generation of TRPV3 agonist carvacrol-induced hair growth inhibition in C57BL/6 mice. Bottom panel, mice (7-8 weeks) were shaved and given different compounds topically once a day until hair grew out, images of hair growth were recorded every 3 days. Scale bar, $500 \mu \mathrm{m}$. (B) Summary from (A) for hair length randomly plucked and measured $(n=8)$ at different time intervals $(9,12,15,18$, and 21 day) after topical application of different concentrations of TRPV3 agonist carvacrol (Car) and antagonist forsythoside B. Data are presented as the mean \pm S.D. (****P $<0.0001$, by two-way ANOVA, followed by Dunnett's test.) (C) A self-comparison group between the left and right side of the skin after topical application of $191.1 \mathrm{mM}$ carvacrol and vehicle (50\% ethanol), respectively, once a day, and images for hair growth were recorded every 3 days. Scale bar, $500 \mu \mathrm{m}$. (D) Summary from (C) for comparison of hair length randomly plucked $(n=8)$ between left $(\mathrm{L})$ and right $(\mathrm{R})$ side of the skin. Data are presented as the mean \pm S.D. ( $* * * * P<0.0001$, by two-way ANOVA, followed by Sidak's test.)

carvacrol $(300 \mu \mathrm{M})$ significantly reversed ORS cell death induced by carvacrol in a dose-dependent manner (Fig. 2C). Forsythoside $\mathrm{B}$ at 3 and $100 \mu \mathrm{M}$ reduced ORS cell death ratio to $23.07 \% \pm 2.45 \%(P<0.05)$ and $13.78 \% \pm 2.57 \%(P<$ $0.0001)$, respectively, compared with the cell death ratio of $31.18 \% \pm 2.62 \%$ for $300 \mu \mathrm{M}$ carvacrol alone (Fig. 2D).
We also determined the cell viability ratio in ORS cells transfected with TRPV3 shRNA. Transfecting TRPV3 shRNA resulted in a significant reduction of TRPV3 protein expression in ORS cells (Fig. 2E) and significantly reversed the carvacrolinduced effect by increasing ORS cell viability to $78.8 \% \pm$ $3.3 \%$ from $40.8 \% \pm 3.8 \%$ (Fig. $2 \mathrm{~F}$ ). These results demonstrated

TABLE 1

Pharmacological activation of TRPV3 by carvacrol causes delay of hair growth in mice Mean \pm S.D. $n=8 .{ }^{*} p<0.05, *^{* *} p<$ $0.01, * * * p<0.001, * * * * p<0.0001$, by one-way ANOVA, followed by Dunnett's test

\begin{tabular}{lccc}
\hline \multicolumn{1}{c}{ Group } & Time for Pink Skin Turning to Black & Time for Black Skin to Hair Growth & Time to Full Hair Growth \\
\hline & Day & Day & Day \\
Control & $8.0 \pm 0.6$ & $3.0 \pm 0.5$ & $9.0 \pm 0.4$ \\
$50 \%$ Ethanol & $8.0 \pm 0.6$ & $3.0 \pm 0.7$ & $9.0 \pm 0.6$ \\
$0.3 \mathrm{mM} \mathrm{FB}$ & $7.0 \pm 0.4$ & $2.0 \pm 0.5$ & $8.0 \pm 0.6$ \\
$19.1 \mathrm{mM} \mathrm{Car}$ & $9.0 \pm 0.9$ & $4.0 \pm 0.7$ & $9.0 \pm 0.6$ \\
$63.7 \mathrm{mM} \mathrm{Car}$ & $10.0 \pm 1.2^{* *}$ & $4.0 \pm 0.8^{*}$ & $10.0 \pm 0.9$ \\
$191.1 \mathrm{mM} \mathrm{Car}$ & $11.0 \pm 1.8^{* * * *}$ & $5.0 \pm 0.9^{* * *}$ & $10.0 \pm 1.1^{* *}$ \\
\hline
\end{tabular}



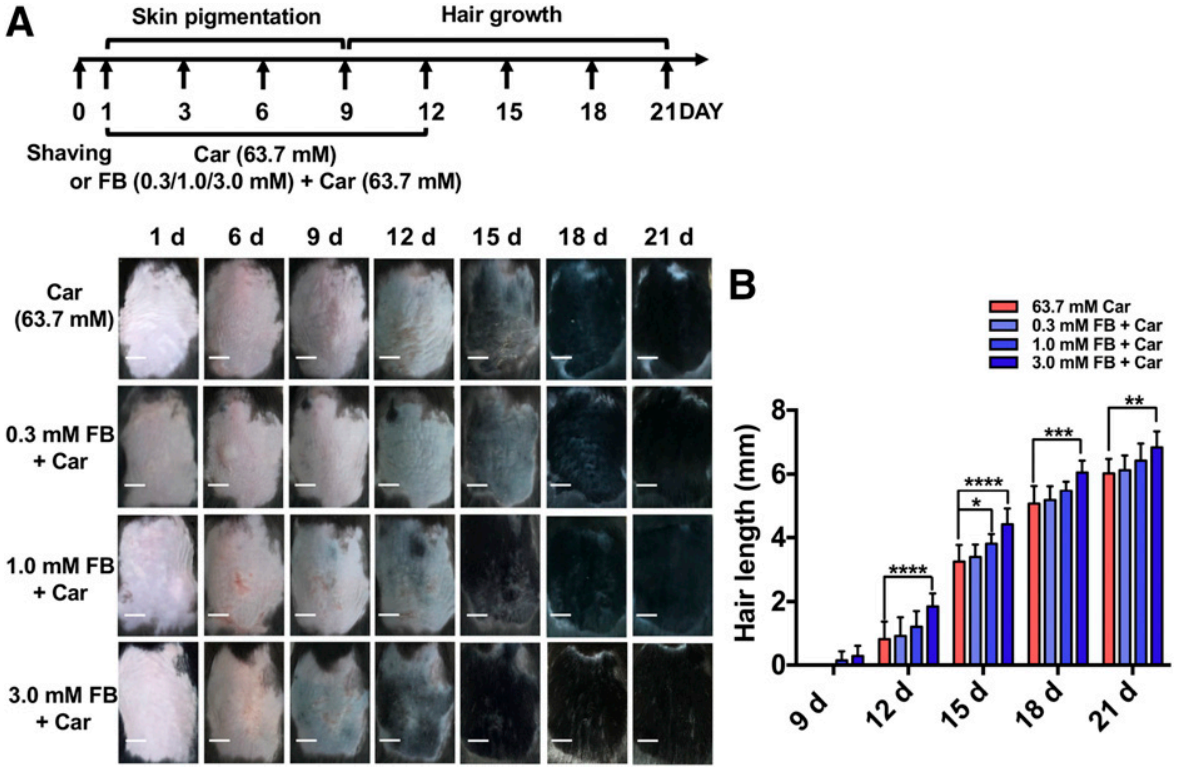

Fig. 4. Inhibition of TRPV3 by forsythoside B attenuates carvacrol-induced hair growth inhibition in mice. (A) Top panel, a schematic drawing for generation of TRPV3 agonist carvacrol-induced hair growth inhibition and treatment with $\mathrm{FB}$ in $\mathrm{C} 57 \mathrm{BL} / 6$ mice. In bottom panel, mice (7-8 weeks) were shaved and intradermal injections of forsythoside $\mathrm{B}$ in different concentrations $(0.3,1,3 \mathrm{mM}$ in $50 \mu \mathrm{l})$ into the dorsal area of skin 30 minutes before topical application of carvacrol $(63.7 \mathrm{mM})$ once a day until hair grew out, images were recorded every 3 days. Scale bar, $500 \mu \mathrm{m}$. (B) Summary from (A) for hair length randomly plucked and measured $(n=8)$ at different time intervals $(9,12,15,18$, and 21 day) after topical application or coapplication of different concentrations of TRPV3 agonist carvacrol (Car) and antagonist forsythoside B. Data are presented as the mean \pm S.D. $(* P<$ $0.05 ; * * P<0.01 ; * * * P<0.001 ; * * * * P<0.0001$, by two-way ANOVA, followed by Dunnett's test.) that pharmacological activation of TRPV3-induced ORS cell death and inhibition of overactive TRPV3 by inhibitor forsythoside B or shRNA reversed the cell death, demonstrating a critical role for TRPV3 in regulation of ORS cell viability.

Time-Dependent Delay of Hair Growth by Agonist Carvacrol-Induced Activation of TRPV3. To examine the impact of TRPV3 modulation on hair growth, we carried out topical applications of different concentrations of carvacrol $(19.1,63.7$, and $191.1 \mathrm{mM}$ in $150 \mu \mathrm{l})$ to the shaved dorsal area $(2.5 \times 3.5 \mathrm{~cm})$ of the skin once a day (Fig. 3A, top panel, $n=8$ ), and evaluated the time-dependent hair growth by observing the skin black pigmentation and hair growth (Fig. 3A). Black pigmentation in the skin is considered a transition from telogen to anagen phase (Paus and Cotsarelis, 1999; Paus and Foitzik, 2004). We observed the three sequential stages of hair growth: pink skin turning black, black skin growing hair, and full hair (Fig. 3A; Table 1). Topical applications of TRPV3 agonist carvacrol (19.1, 63.7, and $191.1 \mathrm{mM}$ ) caused a concentration-dependent delay of pink skin turning black from day 9 to day 12 (Fig. 3A; Table 1), compared with the blank control (50\% ethanol) or the $0.3 \mathrm{mM}$ forsythoside B group that exhibited a visible black coloration on day 9 (Fig. 3A). Likewise, topical applications of carvacrol $(19.1,63.7$, and $191.1 \mathrm{mM})$ also caused a concentrationdependent delay in black skin starting to grow hairs from day 12 to day 15 (Fig. 3A), compared with blank control or forsythoside $\mathrm{B}$ group that started to grow hairs on day 12 (Fig. 3A; Table 1). For measurement of hair length, applications of carvacrol $(19.1,63.7$, and $191.1 \mathrm{mM})$ resulted in concentration-dependent reduction of hair length, compared with blank control or $0.3 \mathrm{mM}$ forsythoside B group (Fig. 3, $\mathrm{A}$ and $\mathrm{B})$. These results indicate that activation of TRPV3 by carvacrol inhibits hair growth in both time- and concentrationdependent manner.

We also measured the hair growth by comparing the left and right side of dorsal skin in a same mouse after topical applications of $191.1 \mathrm{mM}$ carvacrol on the left and $50 \%$ ethanol on the right as control. As shown in Fig. 3C, the left side of dorsal skin treated with $191.1 \mathrm{mM}$ carvacrol only started to become dark on day 12, and hair began to grow on day 15. In contrast, the right side of the skin treated with $50 \%$ ethanol started to become dark on day 9 and hair already grew on day 12 , indicating at least a 3-day delay of hair growth on the left part of the skin treated with TRPV3 agonist carvacrol. To further confirm hair growth inhibition by carvacrol, we measured the length of hairs plucked from mice on days 9 , $12,15,18$, and 21 . The hair length on the left dorsal skin was significantly shorter than the right side in a time-dependent manner (Fig. 3D, $n=8, P<0.0001$ ).

Inhibition of TRPV3 by Natural Inhibitor Forsythoside B Reversed Hair Growth Inhibition Induced by Carvacrol. To further confirm the effect of TRPV3 activation on hair growth inhibition, we carried out intradermal injection of forsythoside B at different concentrations $(0.3,1,3 \mathrm{mM}$ in $50 \mu \mathrm{l})$ into mouse dorsal skin 30 minutes before topical application of carvacrol $(63.7 \mathrm{mM})$ onto the same area. Compared with the group of carvacrol $(63.7 \mathrm{mM})$ alone, intradermal injections of forsythoside B together with carvacrol $(63.7 \mathrm{mM})$ reversed hair growth inhibition by shortening

TABLE 2

Reversal of TRPV3 agonist carvacrol-induced inhibition of hair growth by inhibitor forsythoside B Mean \pm S.D. $n=8$. $p<$ 0.05, by one-way ANOVA, followed by Dunnett's test

\begin{tabular}{cccr}
\hline Group & Time for Pink Skin to Black & Time for Black Skin to Hair Growth & Time to Full Growth of Hair \\
\hline & Day & Day & Day \\
63.7 mM Car & $10.0 \pm 0.9$ & $4.0 \pm 0.7$ & $10.0 \pm 1.0$ \\
Car +0.3 mM FB & $9.0 \pm 1.8$ & $4.0 \pm 0.7$ & $10.0 \pm 1.1$ \\
Car +1.0 mM FB & $9.0 \pm 1.2$ & $4.0 \pm 0.6$ & $9.0 \pm 0.8$ \\
Car +3.0 mM FB & $7.0 \pm 0.7^{*}$ & $3.0 \pm 0.5^{*}$ & $9.0 \pm 0.6$ \\
\hline
\end{tabular}


A

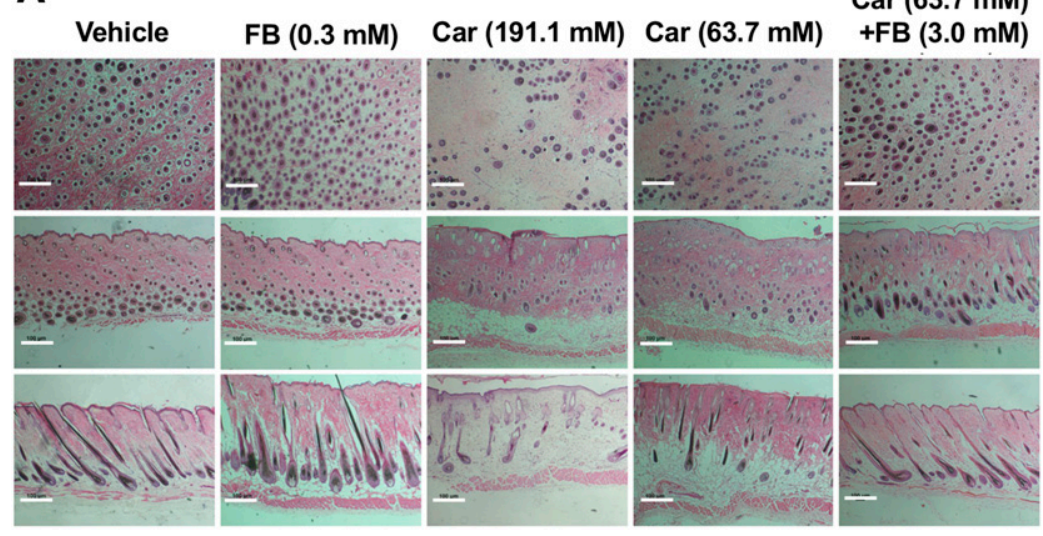

B

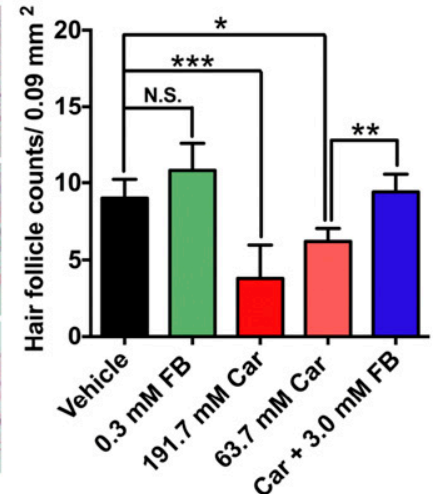

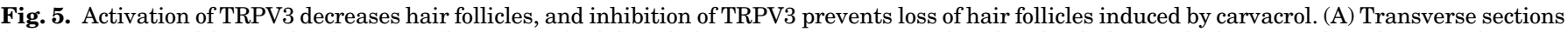

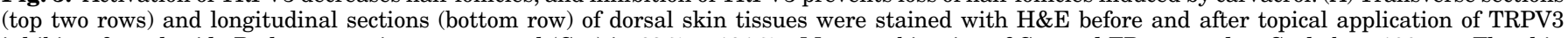

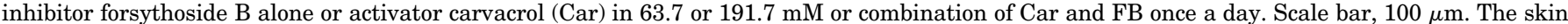

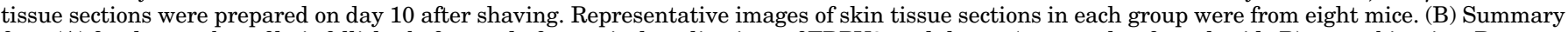

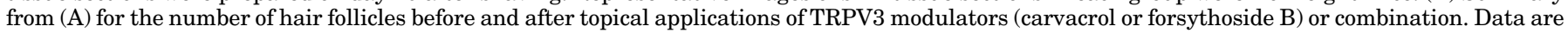
presented as the mean \pm S.D. (N.S., no significance, $* P<0.05 ; * * P<0.01$; ***P<0.001, by one-way ANOVA followed by Bonferroni's test.)

the time of pink skin turning to black (reduced to 7 days from 10 days), and the time from black skin to hair growth (reduced to 3 days from 4 days) and to full growth of hair (reduced to 9 days from 10 days) in both concentration-dependent and time-dependent fashions (Fig. 4A; Table 2). Likewise, the length of hair in carvacrol $(63.7 \mathrm{mM})$-treated group was significantly shorter than the blank control or forsythoside B groups (Fig. 4B). These results demonstrated that pharmacological activation of TRPV3 by carvacrol inhibited hair growth, and inhibition of TRPV3 by forsythoside $\mathrm{B}$ reversed hair growth inhibition induced by carvacrol.

Reversal of TRPV3 Activation-Mediated Decrease of Hair Follicles by Inhibitor Forsythoside B. Anagen phase is critical for hair follicle development (Ogawa and Hattori, 1983), and the telogen phase is the resting phase of hair follicle (Datta et al., 2009). For examination of TRPV3 modulation of the hair growth cycle, H\&E staining of skin tissue sections was performed. In the vehicle- or forsythoside B-treated groups, hair follicles were densely distributed in transverse tissue sections and extended in a fixed direction of growth in longitudinal tissue sections (Fig. 5A), compared with sparse hair follicles and their irregular growth directions in either 63.7- or 191.1-mM carvacrol-treated groups (Fig. 5A). Likewise, dermal injection of forsythoside B (3 mM in $50 \mu \mathrm{l}$ ) significantly reversed the loss of hair follicles caused by topical application of $63.7 \mathrm{mM}$ carvacrol (Fig. $5 \mathrm{~A}, n=8$, $P<0.01)$. Further analysis confirmed that the number of hair follicles per area $\left(0.09 \mathrm{~mm}^{2}\right)$ in carvacrol-treated groups was significantly lower than vehicle- or forsythoside B-treated groups (Fig. 5B). These results indicated that inhibition of TRPV3 by forsythoside B reversed the decrease of hair follicles induced by TRPV3 agonist carvacrol.

\section{Discussion}

On the basis of our previous results and those of others that gain-of-function mutations in TRPV3 cause genetic Olmsted syndrome characterized by alopecia in humans and hair loss in rodents, in this study, therefore, we tested the hypothesis that TRPV3 in the keratinocyte of the skin is essential for hair development and growth. Using approaches combining biochemistry, cell biology, immunohistochemistry, and in vivo models, we examined the effect of TRPV3 channel modulation on hair follicles and hair growth. Our findings show that pharmacological activation of TRPV3 by carvacrol inhibits hair growth in mice by suppressing hair shaft elongation and reducing hair follicles. Pharmacological activation of TRPV3 also leads to death of ORS cells, and specific inhibition of TRPV3 by the natural compound forsythoside B reverses hair growth inhibition and death of ORS induced by carvacrol. Mechanistically, we propose that activation of TRPV3 results in an excessive increase of intracellular calcium in ORS cells critical for maintaining the intact structure of hair follicles, thus leading to ORS cell death via activation of an unidentified $\mathrm{Ca}^{2+}$-dependent kinase, and also inhibition of hair growth (Fig. 6).

Overactive TRPV3 has been shown to be involved in dermatitis and hair loss. In WBN/Kob-Ht rats or DS-Nh mice, the spontaneous gain-of-function mutation (G573C or G573S) of the TRPV3 gene causes cutaneous inflammation, itchiness, and hair loss (Asakawa et al., 2006). We and others have previously identified the gain-of-function mutations (G573C, G573S, and $\mathrm{W} 692 \mathrm{G}$ ) in the same gating area of the TRPV3 channel gene from humans that lead to genetic disease of Olmsted syndrome characterized by dermatitis, palmoplantar keratosis, severe itching, and alopecia (Lai-Cheong et al., 2012; Lin et al., 2012; Choi et al., 2018). Although genetically linked between hair loss and dermatitis, whether and how TRPV3 directly affects hair follicles and hair growth still remain largely unknown. This is mostly a result of the significant lack of specific modulators for TRPV3, which has impeded our understanding of TRPV3 channel function in skin pharmacology and pathology. Our recent investigations demonstrate that pharmacological activation of TRPV3 channel function by carvacrol (5-isopropyl-2-methylphenol), a natural skin sensitizer and relatively specific agonist for TRPV3 (over TRPA1), can evoke dermatitis characterized by pruritus in mice (Cui et al., 2018), suggesting the involvement of TRPV3 in hair growth. To verify this notion, we used carvacrol as a tool and tested its effect on skin and hair growth in this 


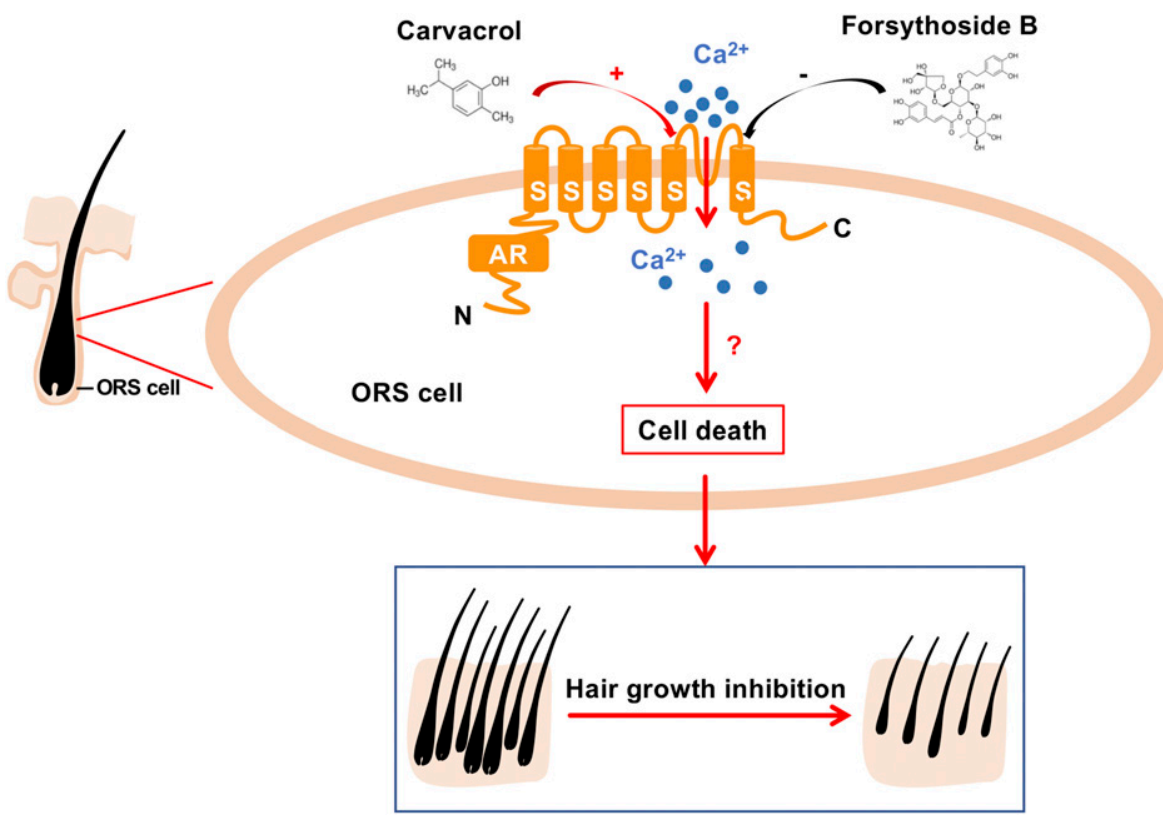

Fig. 6. A proposed mechanism underlying the death for hair follicle ORS cells and hair loss by TRPV3 activation. TRPV3 channels are expressed in the hair follicle ORS cells. Activation of TRPV3 channel by agonist carvacrol or gain-of-function mutation causes an excessive $\mathrm{Ca}^{2+}$ influx that induces ORS cell death via activation of unidentified $\mathrm{Ca}^{2+}$-dependent kinase. Overactive TRPV3 either by carvacrol (or gain-of-function mutations) causes a time- and dose-dependent reduction of hair shaft elongation and a decrease in hair follicles Probably through the induction of ORS cell death in hair follicles, thus leading to inhibition of hair growth. Pharmacological inhibition of overactive TRPV3 by forsythoside B can reverse hair loss.

study. Pharmacological activation of TRPV3 by carvacrol can indeed cause hair loss by suppressing hair shaft elongation and reducing hair follicles. To validate the role of TRPV3 in hair loss, we also took advantage of the selective TRPV3 inhibitor forsythoside $\mathrm{B}$ as a tool in this investigation. Forsythoside B is an active ingredient of Forsythia suspensa fruits and Lamiophlomis rotate leaves commonly used as the traditional Chinese herbal medicines for alleviation of inflammation and detumescence (Jiang et al., 2010; Muluye et al., 2014; Zhu et al., 2014), and it also attenuates dermatitis induced by either pruritogen or TRPV3 agonist carvacrol (Zhang et al., 2019). In this study, we were able to show that inhibition of TRPV3 by natural forsythoside B reverses hair growth inhibition caused by carvacrol and also reduces ORS cell death. These findings provide an evidence for cutaneous TRPV3 that is critical for hair growth, and pharmacological modulation of TRPV3 channel function in the keratinocytes may be beneficial for hair growth, care, hygiene, and cosmetology.

Besides TRPV3, a few other TRP channels have been suggested as playing a role in regulation of hair growth. The immunoreactivity of capsaicin receptor TRPV1 channel has been detected in epidermal hair follicle keratinocytes, sebocytes, mast cells, and dendritic cells (Denda et al., 2001; Bodó et al., 2004). Stimulation of TRPV1 induces apoptosis of ORS keratinocytes and causes the release of proinflammatory cytokines (Bodó et al., 2005; Bíró et al., 2006), and TRPV1 knockout results in a delay in hair follicle cycling (Bíró et al., 2006). Another thermosensitive TRPV4 is also expressed in sebaceous gland cells and ORS keratinocytes (Garcia-Elias et al., 2014; Szabo et al., 2019), and TRPV4 activation can dose dependently inhibit hair shaft elongation in vitro (Szabo et al., 2019). Other transient receptor potential channels, such as transient receptors potential canonical 1 and 4 (TRPC1 and TRPC4), transient receptor potential vanilloid 5 (TRPV5), TRPA1, and transient receptor potential melastatin 8 (TRPM8) have also been reported to be expressed in cutaneous keratinocytes, but whether they play some role in regulation of hair growth remains to be explored (Bezzerides et al., 2004).
Nevertheless, we propose that TRPV3 is essential and critical for regulation of hair growth.

In summary, we have shown that pharmacological activation of TRPV 3 channels inhibits hair growth by inducing death of ORS cells, and inhibition of TRPV3 attenuates hair growth inhibition caused by the overactive channel function. These results demonstrate the critical role of TRPV 3 channel function in hair follicles for regulation of hair growth, and topical inhibition of TRPV3 may represent a promising therapeutic strategy for prevention or treatment of hair follicle-related hair loss or skin disease.

\section{Acknowledgments}

We thank our laboratory members, Q. Zhou, H. Yin, H. Zhang, and Y. Qu for their technical assistance in this study. K.Y. thanks Y. P. Wang for his emotional support.

\section{Authorship Contributions}

Participated in research design: Yan, Sun, K. Wang.

Conducted experiments: Yan, Sun, Liu.

Performed data analysis: Yan, Sun, G. Wang.

Wrote or contributed to the writing of the manuscript: Yan, Sun, $\mathrm{K}$. Wang.

\section{References}

Aijima R, Wang B, Takao T, Mihara H, Kashio M, Ohsaki Y, Zhang JQ, Mizuno A, Suzuki M, Yamashita Y, et al. (2015) The thermosensitive TRPV3 channel contributes to rapid wound healing in oral epithelia. FASEB $J$ 29:182-192.

Asakawa M, Yoshioka T, Matsutani T, Hikita I, Suzuki M, Oshima I, Tsukahara K, Arimura A, Horikawa T, Hirasawa T, et al. (2006) Association of a mutation in TRPV3 with defective hair growth in rodents. J Invest Dermatol 126:2664-2672.

Bang S, Yoo S, Yang TJ, Cho H, and Hwang SW (2010) Farnesyl pyrophosphate is a novel pain-producing molecule via specific activation of TRPV3. J Biol Chem 285: 19362-19371.

Bezzerides VJI, Ramsey IS, Kotecha S, Greka A, and Clapham DE (2004) Rapid vesicular translocation and insertion of TRP channels. Nat Cell Biol 6:709-720.

Bíró T, Bodó E, Telek A, Géczy T, Tychsen B, Kovács L, and Paus R (2006) Hair cycle control by vanilloid receptor-1 (TRPV1): evidence from TRPV1 knockout mice. $J$ Invest Dermatol 126:1909-1912.

Bodó E, Bíró T, Telek A, Czifra G, Griger Z, Tóth BI, Mescalchin A, Ito T, Bettermann A, Kovács L, et al. (2005) A hot new twist to hair biology: involvement of vanilloid receptor-1 (VR1/TRPV1) signaling in human hair growth control. Am J Pathol 166: 985-998.

Bodó E, Kovács I, Telek A, Varga A, Paus R, Kovács L, and Bíró T (2004) Vanilloid receptor-1 (VR1) is widely expressed on various epithelial and mesenchymal cell types of human skin. J Invest Dermatol 123:410-413. 
Borbíró I, Lisztes E, Tóth BI, Czifra G, Oláh A, Szöllosi AG, Szentandrássy N, Nánási PP, Péter Z, Paus R, et al. (2011) Activation of transient receptor potential vanilloid-3 inhibits human hair growth. J Invest Dermatol 131:1605-1614.

Cao X, Yang F, Zheng J, and Wang K (2012) Intracellular proton-mediated activation of TRPV3 channels accounts for the exfoliation effect of $\alpha$-hydroxyl acids on keratinocytes. J Biol Chem 287:25905-25916.

Chen CC and Chuong CM (2012) Multi-layered environmental regulation on the homeostasis of stem cells: the saga of hair growth and alopecia. J Dermatol Sci $\mathbf{6 6}$ : $3-11$.

Cheng X, Jin J, Hu L, Shen D, Dong XP, Samie MA, Knoff J, Eisinger B, Liu ML, Huang SM, et al. (2010) TRP channel regulates EGFR signaling in hair morphogenesis and skin barrier formation. Cell 141:331-343.

Choi JY, Kim SE, Lee SE, and Kim SC (2018) Olmsted syndrome caused by a heterozygous p.Gly568Val missense mutation in TRPV3 gene. Yonsei Med $J$ 59:341-344.

Chung MK, Lee H, Mizuno A, Suzuki M, and Caterina MJ (2004a) 2-aminoethoxydiphenyl borate activates and sensitizes the heat-gated ion channel TRPV3. J Neurosci 24:5177-5182.

Chung MK, Lee H, Mizuno A, Suzuki M, and Caterina MJ (2004b) TRPV3 and TRPV4 mediate warmth-evoked currents in primary mouse keratinocytes. $J$ Biol Chem 279:21569-21575.

Clapham DE (2003) TRP channels as cellular sensors. Nature 426:517-524

Cui TT, Wang GX, Wei NN, and Wang K (2018) A pivotal role for the activation of TRPV3 channel in itch sensations induced by the natural skin sensitizer carvacrol. Acta Pharmacol Sin 39:331-335.

Datta K, Singh AT, Mukherjee A, Bhat B, Ramesh B, and Burman AC (2009) Eclipta alba extract with potential for hair growth promoting activity. J Ethnopharmacol 124:450-456.

Denda M, Fuziwara S, Inoue K, Denda S, Akamatsu H, Tomitaka A, and Matsunaga K (2001) Immunoreactivity of VR1 on epidermal keratinocyte of human skin. Biochem Biophys Res Commun 285:1250-1252.

Garcia-Elias A, Mrkonjić S, Jung C, Pardo-Pastor C, Vicente R, and Valverde MA (2014) The TRPV4 channel. Handb Exp Pharmacol 222:293-319.

Jiang WL, Fu FH, Xu BM, Tian JW, Zhu HB, and Jian-Hou (2010) Cardioprotection with forsythoside $\mathrm{B}$ in rat myocardial ischemia-reperfusion injury: relation to inflammation response. Phytomedicine 17:635-639.

Jordt S-E, McKemy DD, and Julius D (2003) Lessons from peppers and peppermint: the molecular logic of thermosensation. Curr Opin Neurobiol 13:487-492.

Kunz M, Seifert B, and Trüeb RM (2009) Seasonality of hair shedding in healthy women complaining of hair loss. Dermatology 219:105-110.

Lai-Cheong JE, Sethuraman G, Ramam M, Stone K, Simpson MA, and McGrath JA (2012) Recurrent heterozygous missense mutation, p.Gly573Ser, in the TRPV3 gene in an Indian boy with sporadic Olmsted syndrome. Br J Dermatol 167:440-442.

Lin Z, Chen Q, Lee M, Cao X, Zhang J, Ma D, Chen L, Hu X, Wang H, Wang X, et al. (2012) Exome sequencing reveals mutations in TRPV3 as a cause of Olmsted syndrome. Am J Hum Genet 90:558-564.

Moqrich A, Hwang SW, Earley TJ, Petrus MJ, Murray AN, Spencer KS, Andahazy M, Story GM, and Patapoutian A (2005) Impaired thermosensation in mice lacking TRPV3, a heat and camphor sensor in the skin. Science 307:1468-1472.

Müller-Röver S, Handjiski B, van der Veen C, Eichmüller S, Foitzik K, McKay IA, Stenn KS, and Paus R (2001) A comprehensive guide for the accurate classification of murine hair follicles in distinct hair cycle stages. $J$ Invest Dermatol 117:3-15.

Muluye RA, Bian Y, and Alemu PN (2014) Anti-inflammatory and antimicrobial effects of heat-clearing Chinese herbs: a current review. J Tradit Complement Med 4:93-98.

Ogawa H and Hattori M (1983) Regulation mechanisms of hair growth. Curr Probl Dermatol 11:159-170.
Paus R (1998) Principles of hair cycle control. J Dermatol 25:793-802

Paus R and Cotsarelis G (1999) The biology of hair follicles. $N$ Engl $J$ Med 341: 491-497.

Paus R and Foitzik K (2004) In search of the "hair cycle clock": a guided tour. Differentiation 72:489-511.

Peier AM, Reeve AJ, Andersson DA, Moqrich A, Earley TJ, Hergarden AC, Story GM, Colley S, Hogenesch JB, McIntyre P, et al. (2002) A heat-sensitive TRP channel expressed in keratinocytes. Science 296:2046-2049.

Schneider MR, Werner S, Paus R, and Wolf E (2008) Beyond wavy hairs: the epidermal growth factor receptor and its ligands in skin biology and pathology. Am J Pathol 173:14-24.

Slominski A and Paus R (1993) Melanogenesis is coupled to murine anagen: toward new concepts for the role of melanocytes and the regulation of melanogenesis in hair growth. J Invest Dermatol 101 (1 Suppl):90S-97S.

Slominski A, Paus R, Plonka P, Chakraborty A, Maurer M, Pruski D, and Lukiewicz $\mathrm{S}$ (1994) Melanogenesis during the anagen-catagen-telogen transformation of the murine hair cycle. J Invest Dermatol 102:862-869.

Spencer LV and Callen JP (1987) Hair loss in systemic disease. Dermatol Clin 5: $565-570$.

Sperling LC (2001) Hair and systemic disease. Dermatol Clin 19:711-726, ix.

Steinhoff M and Bíró T (2009) A TR(I)P to pruritus research: role of TRPV3 in inflammation and itch. J Invest Dermatol 129:531-535.

Stenn KS and Paus R (2001) Controls of hair follicle cycling. Physiol Rev 81:449-494

Szabo IL, Herczeg-Lisztes E, Szegedi A, Nemes B, Paus R, Bíró T, and Szöllősi AG (2019) TRPV4 is expressed in human hair follicles and inhibits hair growth in vitro. J Invest Dermatol 139:1385-1388.

Szántó M, Oláh A, Szöllősi AG, Tóth KF, Páyer E, Czakó N, Pór Á, Kovács I, Zouboulis CC, Kemény L, et al. (2019) Activation of TRPV3 inhibits lipogenesis and stimulates production of inflammatory mediators in human sebocytesa putative contributor to dry skin dermatoses. J Invest Dermatol 139:250-253.

Szöllősi AG, Vasas N, Angyal Â, Kistamás K, Nánási PP, Mihály J, Béke G, HerczegLisztes E, Szegedi A, Kawada N, et al. (2018) Activation of TRPV3 regulates inflammatory actions of human epidermal keratinocytes. J Invest Dermatol 138: 365-374.

Vogt-Eisele AK, Weber K, Sherkheli MA, Vielhaber G, Panten J, Gisselmann G, and Hatt $\mathrm{H}$ (2007) Monoterpenoid agonists of TRPV3. $\mathrm{Br} J$ Pharmacol 151: 530-540.

Wang G and Wang $\mathrm{K}(2017)$ The $\mathrm{Ca}^{2+}$-permeable cation transient receptor potential TRPV3 channel: an emerging pivotal target for Itch and skin diseases. $M o l$ Pharmacol 92:193-200.

Xu H, Delling M, Jun JC, and Clapham DE (2006) Oregano, thyme and clove-derived flavors and skin sensitizers activate specific TRP channels. Nat Neurosci 9: 628-635.

Zhang H, Sun X, Qi H, Ma Q, Zhou Q, Wang W, and Wang K (2019) Pharmacological inhibition of the temperature-sensitive and $\mathrm{Ca}^{2+}$-permeable transient receptor potential vanilloid TRPV3 channel by natural forsythoside B attenuates pruritus and cytotoxicity of keratinocytes. J Pharmacol Exp Ther 368:21-31.

Zhu B, Gong N, Fan H, Peng CS, Ding XJ, Jiang Y, and Wang YX (2014) Lamiophlomis rotata, an orally available Tibetan herbal painkiller, specifically reduces pain hypersensitivity states through the activation of spinal glucagon-like peptide-1 receptors. Anesthesiology 121:835-851.

Address correspondence to: Dr. KeWei Wang, Department of Pharmacology, School of Pharmacy, Qingdao University, 38 Dengzhou Road, Qingdao 266021, China. E-mail: wangkw@qdu.edu.cn 